\title{
Adiciones al hormigón basadas en grasas orgánicas
}

Cement and Lime Manufacture, n 6, noviembre 1969, págs. 107-114

En unas reuniones celebradas en Milán sobre "El sebo y sus derivados", el Dr. D. M. Doty presentó un trabajo sobre "Materiales derivados de la grasa en la composición del cemento". La Fundación para Investigación de Grasas y Proteínas de Des Plaines, Illinois, USA, y de la que es Director el autor de este trabajo, está continuamente buscando nuevas salidas para las grasas de aves y animales como parte de su actividad investigadora.

La investigación ha resultado en el desarrollo de tres materiales derivados de las grasas que muestran ser prometedores en la composición del cemento. Estos son:

a) ácido carboxiesteárico como un agente oclusor de aire para el hormigón;

b) silanos derivados de sebos $u$ otras grasas $u$ olefinas que tienen excelentes propiedades repelentes al agua cuando se aplican como recubrimiento a morteros $u$ hormigones de cemento;

c) una composición grasa-inorgánica en forma de emulsión o pasta que puede añadirse a las mezclas de cemento para mejorar la trabajabilidad, aumentar la resistencia e impartir un alto grado de resistencia al agua a las estructuras de hormigón.

\section{AGENTE OCLUSOR DE AIRE DERIVADO DEL SEBO}

Corrientemente los agentes oclusores de aire comerciales incluyen una serie de productos químicos, pero con mucho la que más prevalece es la resina vinsol. Estos agęntes tienen ciertas limitaciones. Requieren un ajuste de concentración con las distintas mezclas de cemento, la cantidad de aire ocluido es muy sensible a la concentración de los productos químicos y el comportamiento es relativamente pobre con composiciones de cemento que contienen cenizas de combustible pulverizado.

Sobre la base de las características químicas conocidas de los buenos agentes oclusores de aire, pareció que el ácido 9-carboxiesteárico (9-CSA) sería un compuesto satisfactorio para este fin. En consecuencia, se sintetizó una cierta cantidad de este compuesto químico a partir de ácido oleico y se ensayó. Al emplear 9-CSA, se encontró que la cantidad de aire ocluido no variaba tanto con la concentración como ocurre con los materiales empleados corrientemente (fig. 1). Esto es importante desde el punto de vista práctico porque es difícil de obtener mezclas de hormigón completamente uniformes.

El hormigón producido empleando 9-CSA cumplía las normas ASTM para el hormigón con aire ocluido, en cuanto a su resistencia (tabla 1). 
T A B L A 1

Influencia de los agentes oclusores de aire sobre las caracteristicas del cemento

\begin{tabular}{|l|c|c|c|c|}
\hline & $\begin{array}{c}\text { Ninguna } \\
\text { adición }\end{array}$ & $\begin{array}{c}\text { Adición de } \\
\text { referencia }\end{array}$ & 9-CSA & \\
\hline Aire ocluido & 1,77 & 5,30 & 5,90 & $\%$ \\
Resistencia a compresión & 340 & 310 & 295 & $\mathrm{kp} / \mathrm{cm}^{2}$ \\
Resistencia a flexión & 46 & 45 & 44 & $\mathrm{kp} / \mathrm{cm}^{2}$ \\
Resistencia de agarre & 18 & 22 & 21 & $\mathrm{kp} / \mathrm{cm}^{2}$ \\
\hline
\end{tabular}

Cuando se examinaron microscópicamente muestras pulidas de hormigón con aire ocluido preparado con 9-CSA, se notó que las burbujas de aire eran pequeñas y uniformemente distribuidas, lo que es necesario para una buena resistencia al hielo-deshielo. El hormigón que contenía 9-CSA se ensayó en su resistencia al hielo-deshielo por los procedimientos ASTM prescritos y se encontró que era equivalente al preparado con uno de los mejores productos disponibles en el mercado (fig. 2).
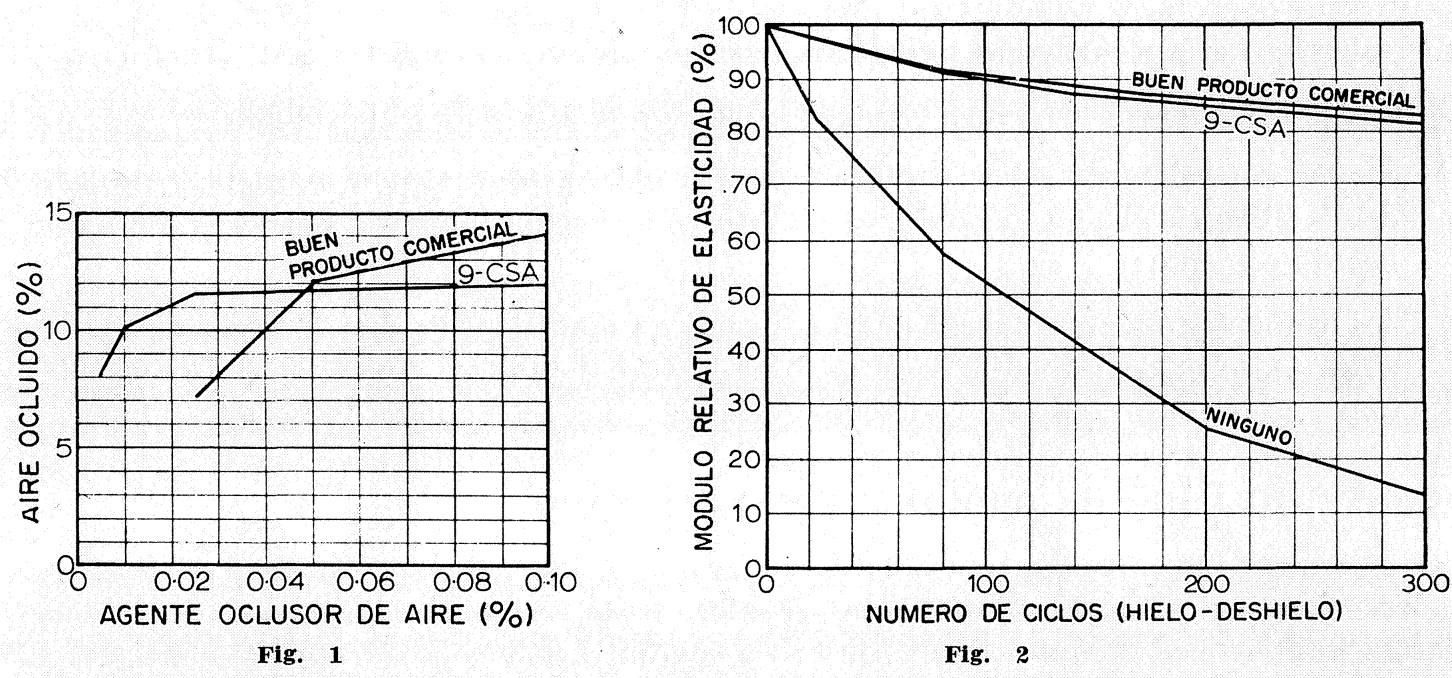

Las ventajas del 9-CSA como un agente oclusor de aire para el hormigón parecen ser que su comportamiento no está estrechamente unido a su concentración, que hay buen comportamiento con hormigón de cenizas de combustible y que el costo es competitivo con los agentes oclusores de aire comerciales puesto que sólo se requiere la mitad de su concentración aproximadamente.

\section{SILANOS DERIVADOS DE GRASAS COMO REVESTIMIENTO REPELENTE AL AGUA}

Puesto que la mayoría de las grasas, particularmente las grasas animales altamente saturadas, son perfectamente hidrofóbicas, deben ser buenos agentes impermeabilizantes pa- 
ra el hormigón si pueden ser fuertemente fijados en el mismo. El único grupo reactivo en las grasas neutras son los enlaces dobles presentes, lo que es necesario para situar el grupo de anclaje en estas posiciones. El grupo triclorosil sería un anclaje útil:

$$
\begin{aligned}
& \mathrm{R}-\mathrm{CH}=\mathrm{CH}-\mathrm{R}^{\prime}+\mathrm{HSiCl}_{3} \stackrel{\mathrm{UV}}{\longrightarrow} \longrightarrow \\
& \mathrm{R}-\mathrm{CH}_{2}-\underset{\mathrm{SiCl}_{3}}{\mathrm{CH}-\mathrm{R}^{\prime}}+\mathrm{R}-\underset{\mathrm{SiCl}_{3}}{\mathrm{CH}}-\mathrm{CH}_{2}-\mathrm{R}^{\prime} \\
& 2 \mathrm{R}-\mathrm{CH}=\mathrm{CH}-\mathrm{R}^{\prime}+\mathrm{H}_{2} \mathrm{SiCl}_{2} \stackrel{\mathrm{UV}}{\longrightarrow} \\
& \mathrm{R}-\mathrm{CH}_{2}-\underset{\mathrm{SiCl}_{2}}{\mathrm{CH}} \text {. } \\
& \mathrm{R}-\mathrm{CH}_{2}-\stackrel{\mathrm{CH}}{\mathrm{SiCl}} \mathbf{\mathrm { R }} \text {, ETC. }
\end{aligned}
$$

Clorosilanos con al menos un átomo de hidrógeno pueden reaccionar con las olefinas como se muestra en la figura 3 . Además puede surgir la hidrólisis de los enlaces $\mathrm{Si}-\mathrm{Cl}$, para producir compuestos siloxy como se muestra en la figura 4. De particular interés es la reacción con un hidroxilicato, tal como puede presentarse en la porción de cemento del hormigón:

$$
\begin{aligned}
& \mathrm{R}_{3} \mathrm{SiCl}+\mathrm{H}_{2} \mathrm{O} \longrightarrow \mathrm{R}_{3} \mathrm{Si}-\mathrm{OH} \stackrel{\mathbf{R}_{3} \mathrm{SiCl}}{\longrightarrow} \mathrm{R}_{3} \mathrm{SiOSiR}_{3} \\
& \mathrm{R}_{3} \mathrm{SiCl}+(\mathrm{SILICATE}-0)_{3} \mathrm{Si}-\mathrm{OH} \longrightarrow \\
& \mathrm{R}_{3} \mathrm{Si}-\mathrm{O}-\mathrm{Si}(-\mathrm{O}-\mathrm{SILICATE})_{3} \\
& \mathrm{R}_{3} \mathrm{SiCl}+\mathrm{R}^{\prime} \mathrm{OH} \longrightarrow \mathrm{R}_{3} \mathrm{SiOR}^{\prime} \\
& \stackrel{\mathrm{O}}{\mathrm{R}} \mathrm{SiCl}+\mathrm{R} \text { "C }-\mathrm{OH} \longrightarrow \mathrm{R}_{3} \mathrm{SiOC}-\mathrm{R}^{\prime \prime}
\end{aligned}
$$

Fig. 4.-Reacción de silanos a partir de compuestos siloxy.

Si ocurren de hecho estas reacciones, el tipo de resultados se representa esquemáticamente en la figura 5, en la que el sebo triclorosilanado (designado TCST) se une al hormigón por reacción con grupos hidroxilicato. El polímero tridimensional con sus diversos grupos hidrofóbicos se formaría in situ para rellenar los poros del hormigón y resistir la penetración de agua.

El TCST se prepara como sigue: Una solución eteretílica de sebo se agita con exceso de triclorosilano en un recipiente de cuarzo en atmósfera de nitrógeno mientras se expone a la luz ultravioleta. El exceso de silano y éter se separa con presión reducida, quedando un líquido incoloro y siruposo. El producto obtenido contiene menos del $10 \%$ de olefinas sin reaccionar y muestra una relación cloro/silicio de 2,7 (el valor teórico es 3 ). Debe señalarse que debe emplearse el sebo seco con un contenido bajo de ácidos libres para prevenir reacciones secundarias durante la síntesis.

Cuando se empleó una solución de TCST al $15 \%$ en keroseno, para revestir el cemento portland hidratado, el análisis térmico diferencial mostró que el TCST creaba de hecho un enlace con el cemento (fig. 6). Esto queda señalado por una desviación en el pico a 
$250^{\circ} \mathrm{C}$, asociado con agua de hidratación y una reducción y desviación del pico del hidróxido cálcico entre $550^{\circ}$ y $600^{\circ} \mathrm{C}$.

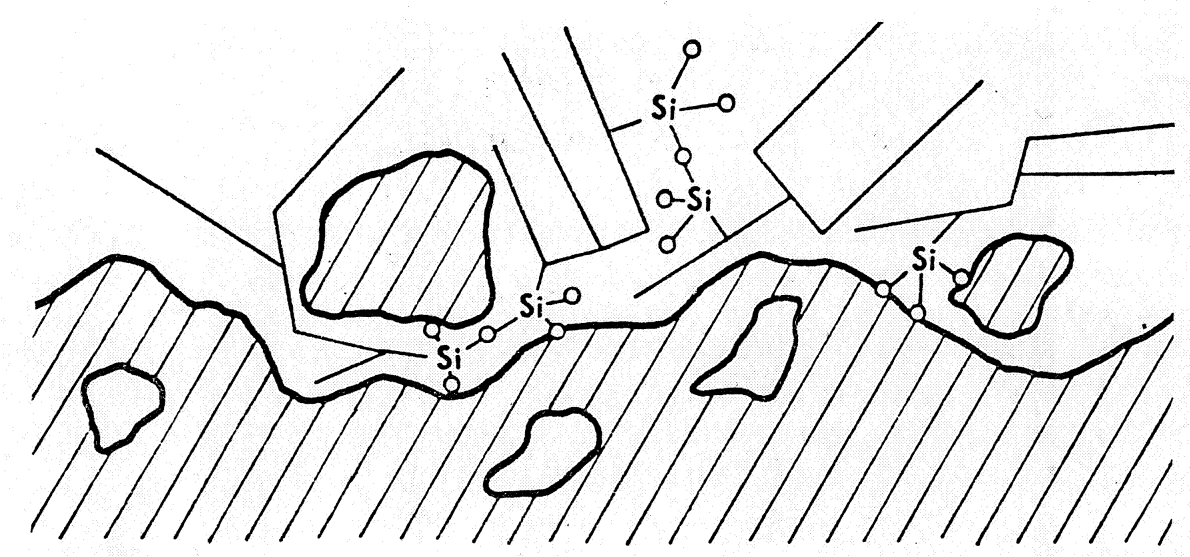

Fig. 5

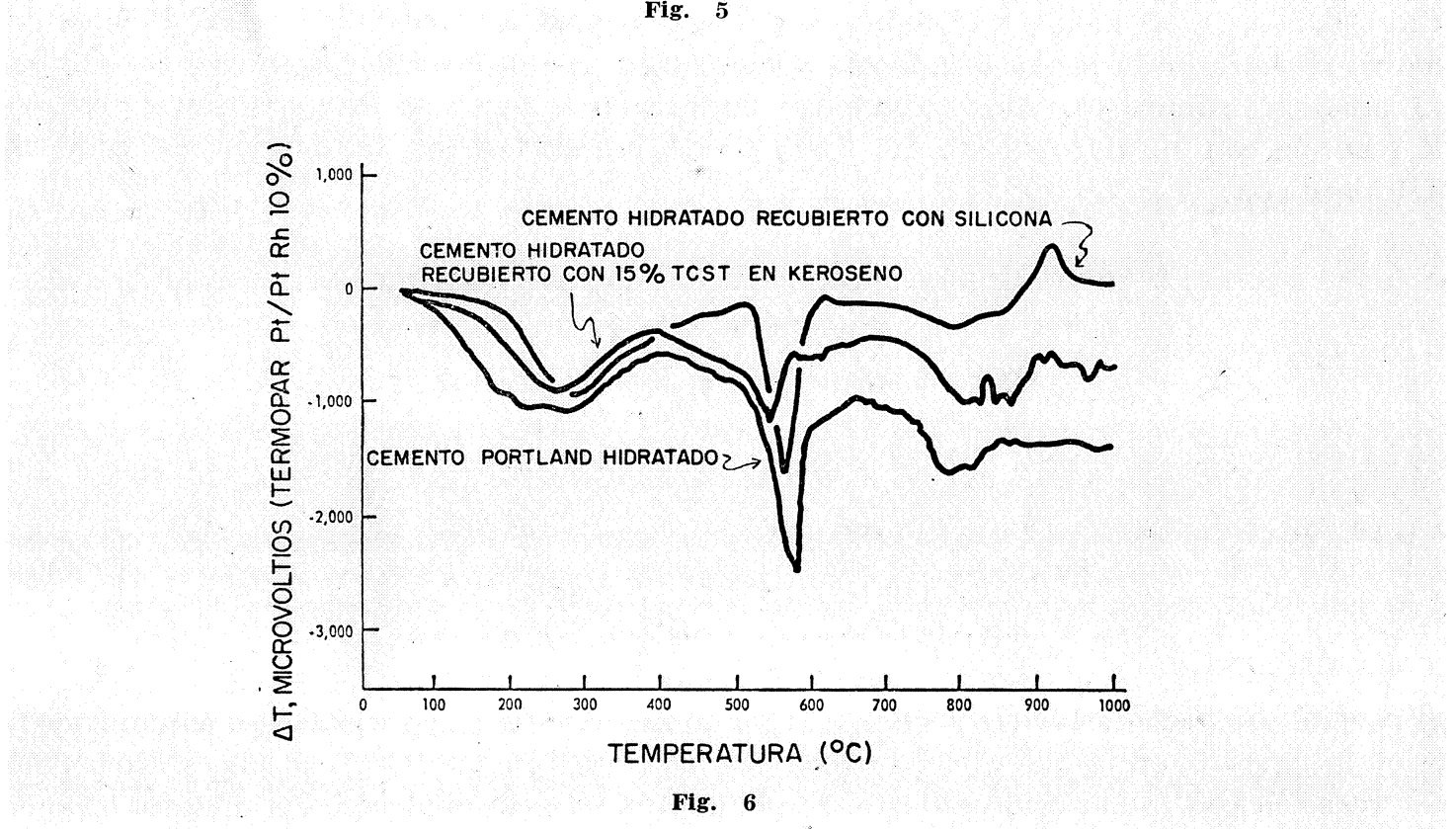

Las muestras de hormigón recubiertas con TCST y mantenidas 3 meses en el laboratorio sumergidas en agua eran mucho más hidrófugas que muestras sin revestir o muestras revestidas con silicona o ácido oleico (fig. 7). Similares resultados se obtenían con probetas expuestas durante 3 meses a un ambiente continuo de niebla y secado e inmersión en agua, aunque es justo reconocer que el ácido oleico era tan efectivo como el TCST bajo estas condiciones (fig. 8).

Otras olefinas silanadas también mostraron buenas características repelentes al agua al emplearse como agentes de revestimiento en el hormigón (fig. 9). Es interesante señalar que ninguno de estos materiales era apreciablemente distinto del TCST, excepto los derivados de aceite de linaza, que eran claramente inferiores. Salvo estos últimos, los deri- 
vados de olefinas eran todos superiores a la preparación de silicona comercial, que resultó totalmente inefectiva bajo las condiciones del ensayo.
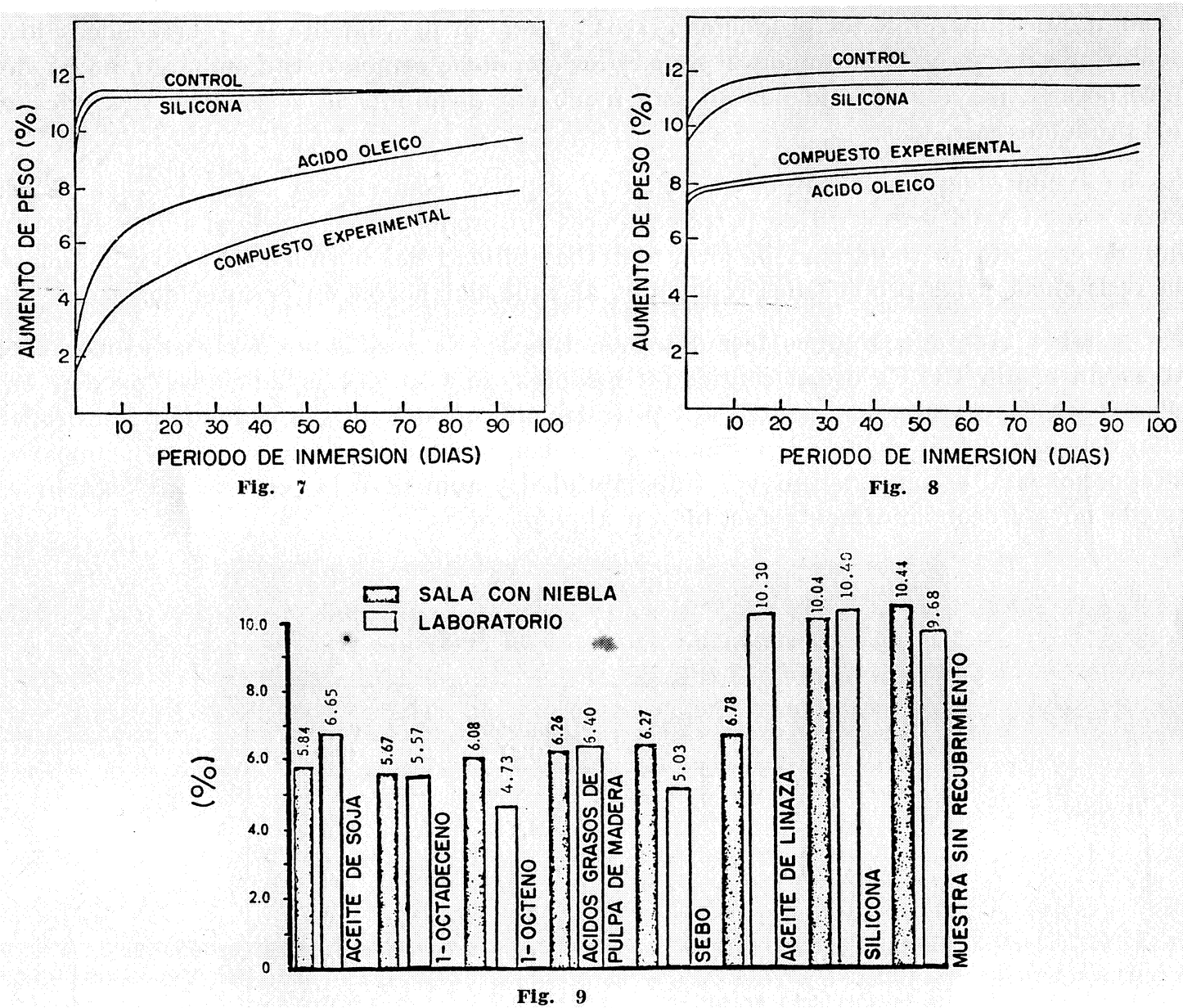

También es significativo que el costo de materias primas para el sebo silanado era apreciablemente más bajo que los costos de materias primas para otras olefinas silanadas (tabla 2). Aunque no se han estimado costos para la producción comercial de TCST, parece claro que el costo de la solución repelente al agua que contiene TCST será menor de la mitad de las formulaciones de silicona ahora disponibles en el mercado.

T A B L A 2

Costo de materias primas para derivados silanados de diferentes olefinas

\begin{tabular}{|l|c|c|c|}
\hline \multicolumn{1}{|c|}{ olefinas } & $\begin{array}{c}\text { Precio } \\
\text { (Ptas.) }\end{array}$ & $\begin{array}{c}\text { Indice de yodo } \\
\text { aproximado }\end{array}$ & $\begin{array}{c}\text { Costo de materias } \\
\text { primas por kilo } \\
\text { producido (Ptas.) }\end{array}$ \\
\cline { 2 - 4 } Sebo & 4,90 & 51 & 16,80 \\
Aceite de soja & 9,80 & 129 & 30,10 \\
Aceite de linaza & 9,10 & 180 & 33,60 \\
Acidos grasos de aceite de madera & 5,60 & 132 & 27,30 \\
1-Octeno & 7,70 & 226 & 35,70 \\
1-Octadeceno & 7,70 & 101 & 25,20 \\
\hline
\end{tabular}




\section{COMPOSICIONES A BASE DE SEBO PARA MEJORAR MORTEROS Y HORMIGONES}

No es raro entre los fabricantes de cemento portland emplear pequeñas cantidades de productos grasos en la molienda de cemento para controlar el polvo y disminuir el desgaste de maquinaria. Estos productos grasos aumentan ligeramente las propiedades hidrofóbicas de morteros y hormigones preparados con dicho cemento. Sin embargo, no pueden incorporarse mayores cantidades de este modo sin disminuir la resistencia mecánica de los productos acabados.

Se ha encontrado, sin embargo, que el sebo disperso con ciertas sales inorgánicas en agua -o en agua y espíritu de petróleo- crea un producto que puede añadirse al hormigón de cemento portland para mejorar la trabajabilidad del hormigón fresco y aumentar la resistencia y las propiedades repelentes al agua del hormigón endurecido.

En la tabla 3 se dan algunas formulaciones típicas. Es posible preparar adiciones efectivas sin añadir $\mathrm{CO}_{3} \mathrm{Ca}$ o con cantidades menores de $\mathrm{Cl}_{2} \mathrm{Ca}$. Las adiciones pueden emplearse en concentraciones de 0,25 a $2 \%$ (relación producto graso/cemento). La proporción depende del producto en particular y de las propiedades que se deseen impartir. Proporciones altas imparten mayor trabajabilidad y aumentan la repelencia al agua, pero puede no ser económicamente factible en algunos casos.

T A B L A 3

Composición de adiciones en emulsión $y$ pasta

\begin{tabular}{|l|c|c|}
\hline & $\begin{array}{c}\text { Emulsión } \\
(\mathbf{k g})\end{array}$ & $\begin{array}{c}\text { Pasta } \\
(\mathbf{k g})\end{array}$ \\
\hline $\mathrm{SiO}_{2}$ & 11,2 & 30,0 \\
$\mathrm{Ca}(\mathrm{OH})_{2}$ & 2,0 & 5,0 \\
$\mathrm{CaCl}_{2}$ & 2,2 & 10,0 \\
$\mathrm{CaCO}_{3}$ & 1,0 & 1,0 \\
$\mathrm{Producto}$ graso & 2,0 & 20,0 \\
Agua & 25,0 & 20,0 \\
Espíritu mineral & - & 10,0 \\
\hline Sólidos totales & $42 \%$ & $69 \%$ \\
\hline
\end{tabular}

Los componentes inorgánicos de la adición empleada sola, darán una resistencia aumentada casi equivalente a la que resulta del empleo de la adición completa (fig. 10), pero no imparte propiedades agua-repelentes.

Aunque todos los productos grasos, empleados en la adición, dan repelencia al agua, el efecto sobre la resistencia a la compresión es notoriamente diferente (tabla 4). Los resultados sugieren que son importantes el grado de insaturación y la cantidad de ácidos grasos libres presentes. Las grasas animales que contienen de 15 a $20 \%$ de ácidos grasos libres producen los mejores resultados.

Aunque las adiciones que contienen ácidos grasos sólo en proporciones de $0,5 \%$ o menos dan resistencia aumentada, las composiciones de cemento hechas con estas adiciones no 
son muy repelentes al agua (fig. 11). Mayores concentraciones de ácidos grasos dieron buenas propiedades repelentes al agua pero resistencia reducida.

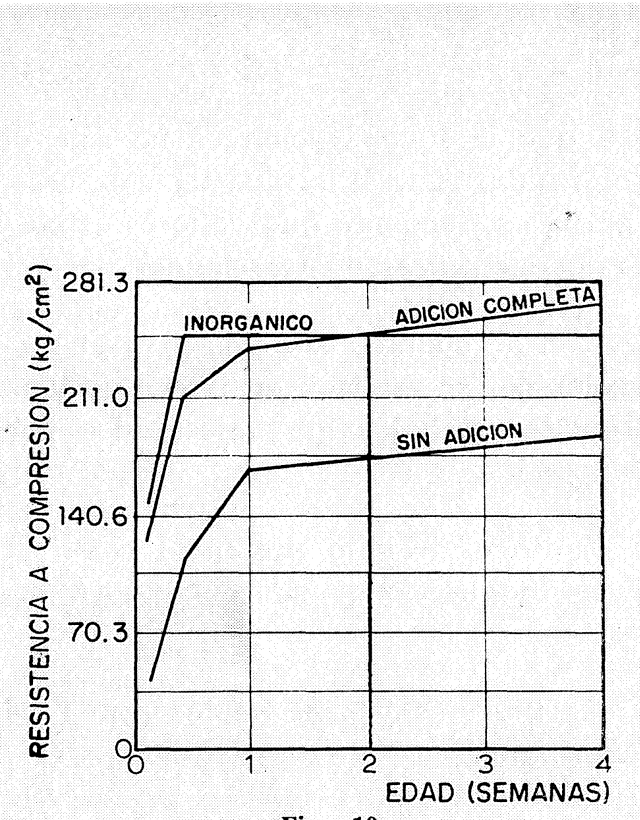

Fig. 10

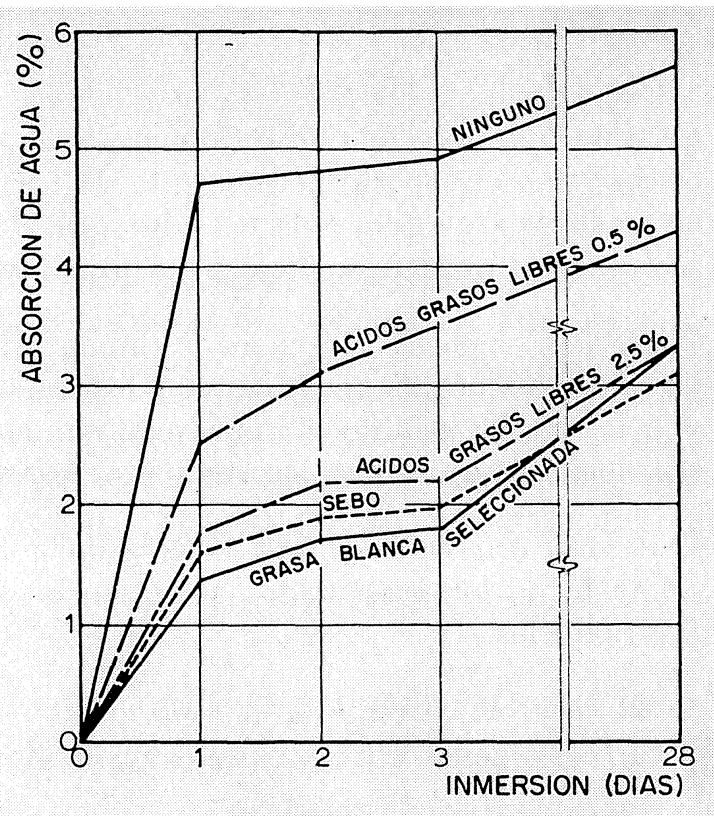

Fig. 11

T A B L A 4

Resistencia a compresión de mortero con diferentes materiales grasos como adición ( $2 \%$ producto graso: 28 días en aire seco)

\begin{tabular}{|l|c|}
\hline \multicolumn{1}{|c|}{ Adición } & $\begin{array}{c}\text { Resistencia } \\
\mathbf{k p} / \mathbf{c m}^{2}\end{array}$ \\
\cline { 1 - 2 } Ninguna & 266 \\
Grasa blanca seleccionada & 239 \\
Sebo n. 1 & 308 \\
Sebo n. 2 & 315 \\
Aceite de soja & 214 \\
Acidos grasos libres $(0,5 \%)$ & 287 \\
Acidos grasos libres $(2,5 \%)$ & 247 \\
\hline
\end{tabular}

\section{ESTADO COMERCIAL}

Se han realizado patentes en Estados Unidos en cuanto a la composición y uso de derivados grasos silanados y en relación con la composición y uso de las adiciones graso-inorgánicas. Patentes similares se han establecido en otros países. Algunos productores de grasas animales de Estados Unidos están autorizados para producir y distribuir las adiciones graso-inorgánicas. También es de esperar a corto plazo, la licencia para la producción y distribución de TCST. El ácido 9-carboxiesteárico es muy similar, en composición química, a otros materiales que se han empleado como agentes oclusores de aire y, por lo tanto, su uso en este aspecto no será probablemente patentable. Sin embargo, se ha hecho un intento para interesar a las compañías en la producción y distribución del 9-CSA como un agente oclusor de aire superior. 